pressure) recommendations were formulated concerning a standardised IAH and ACS monitoring as well as a rational therapeutic management (including medical, interventional and surgical therapy options). While in adults an IAP of $12 \mathrm{mmHg}$ is regarded as dangerous, an IAP of at the latest $10 \mathrm{mmHg}$ must be looked upon as IAH in children. The additional appearance of a new or aggravated organ dysfunction marks the transition into an ACS, whose diagnosis should result in the quickest possible abdominal decompression. If a decompressing laparotomy does not suffice for a durable IAP diminution itself, a prophylactic open-abdomen concept must be considered (syn.: laparostomy). The concept of abdominal perfusion pressure $(\mathrm{APD}=\mathrm{MAP}$ IAP) can facilitate the assessment of the pathogenetic influence of IAH in daily clinical practice.

Discussion In the context of former surveys, paediatric intensivists often justified their widespread uncertainty and restraint with respect to regular IAP measurements and timely introduction of invasive therapy options (if indicated) with the lack of age-related guidelines and definitions. This first publication of paediatric IAP limiting values and management recommendations therefore represents an essential treatment progress and a therapeutic decision support, which should submit a significant reduction in morbidity and mortality of IAH and ACS in children and adolescents.

Conclusion Since the evidence of the underlying literature has to be classified on average as low, well-designed multicenter studies are urgently needed to enable a critical reevaluation of these consensus results.

\section{PS-136 IMPLEMENTING FLEXIBLE BRONCHOSCOPY IN A PICU - A SAFE AND USEFUL TECHNIQUE}

D Vlajnic, N Mueller, N Freudenthal, N Saleh, R Knies. PICU, Children's Hospital, Bonn, Germany

\subsection{6/archdischild-2014-307384.430}

Background and aims In 2011 we started implementing flexible bronchoscopy in our PICU for management of critically ill patients and children with home ventilation. It was our goal to implement this technique as a routine diagnostic an interventional tool and to characterise possible benefits and risks.

Methods This is a prospective study performed in a 10-bed paediatric ICU of a tertiary care children's hospital. All patients who underwent a bronchoscopy in the years 2012-2013 were included in this study. Olympus and Storz bronchoscopes with $2.2 \mathrm{~mm}, 2.8 \mathrm{~mm}$ and $3.8 \mathrm{~mm}$ diameter were used and every procedure was video documented. All procedures were performed by trained paediatric intensive care specialist.

Results 151 procedures were performed in 96 patients. Indications were: Treatment of atelectasis and obstruction (78 of 151 procedures), with improvement in ventilation parameters in 61 of 78 procedures $(78 \%)$; search for airways anatomic pathologies (45 of 151 procedures), with pathological findings in 26 of 45 procedures (58\%); Pneumonia and undetermined lung disease in which cases BAL was preformed (29 of 151 procedures), with changed antimicrobial treatment in 17 of 29 cases $(59 \%)$. We didn't observe any procedure-related mortality or serious complications.

Conclusions Flexible bronchoscopy is a very safe and useful procedure in critical ill infants and children with a variety of diseases, and significantly contributes to their management. In our opinion bronchoscopy should be a routine technique in paediatric intensive care units.

\section{PS-137 LOW BIOCHEMICAL NUTRITIONAL INDICES PREDICT CLINICAL OUTCOMES IN CHILDREN UNDERGOING CARDIAC SURGERY}

G Lim, JH Lee. Paediatric Medicine, KK Women's and Children's Hospital, Singapore, Singapore

10.1136/archdischild-2014-307384.431

Aim To determine whether biochemical nutritional indices predict clinical outcomes in children undergoing cardiac surgery.

Methods Retrospective single centre study between July 2012 and June 2013. Biochemical nutritional indices included serum albumin and total lymphocyte count (TLC) which was used to calculate Onodera's prognostic nutritional index (PNI) $[10 \mathrm{x}$ serum albumin $(\mathrm{g} / \mathrm{dL})+0.005 \mathrm{x}$ TLC $(/ \mathrm{mL})]$. Severity of illness assessed using Paediatric Risk of Mortality (PRISM) III score. Surgery categorised using the risk adjusted classification for congenital heart surgery (RACHS-1) score. Outcomes included hospital mortality, paediatric intensive care unit (PICU) length of stay (LOS) and duration of mechanical ventilation (MV).

Results Total of 31 patients identified. Median age was 2.0 (0.7-41.0) months. Median PRISM III score was 8.0 (5.0-13.0). 14 (45\%) underwent RACHS-1 category 2 surgery and $6(20 \%)$ had ventricular septal defects. Median albumin and TLC were $3.2(2.6-4.2) \mathrm{g} / \mathrm{dL}$ and $4280 \quad(2810-5100) / \mathrm{uL}$ respectively. Median PNI score was 52.7 (41.2-69.7). There was no hospital mortality. Median PICU LOS was 5.0 (3.0-8.0) days. Hypoalbuminaemia $\leq 3.0 \mathrm{~g} / \mathrm{dL}$ associated with longer median PICU LOS (7.0 vs. 4.0 days, $\mathrm{p}=0.016)$ and duration of MV (156 vs. $33 \mathrm{~h}$, $\mathrm{p}=0.007)$. PNI $\leq 58$ associated with longer median PICU LOS (6.0 vs. 3.0 days, $\mathrm{p}=0.041)$. Adjusting for age and RACHS-1 score, for every $1 \mathrm{~g} / \mathrm{dL}$ drop in albumin, PICU LOS increased by 0.5 days $(\mathrm{p}=0.006)$.

Conclusions Hypoalbuminaemia and PNI $\leq 58$ were associated with adverse postoperative outcomes. Future studies to study effect of perioperative aggressive nutrition care on biochemical indices and clinical outcomes.

\section{PS-137a A BRIEF INTERVENTION TO IMPROVE PARENT POST-TRAUMATIC STRESS SYMPTOMS FOLLOWING PAEDIATRIC CRITICAL ILLNESS: A PILOT RANDOMISED CONTROLLED TRIAL}

${ }^{1} \mathrm{LC}$ Als, ${ }^{2} \mathrm{~B}$ Vickers, ${ }^{3} \mathrm{~S}$ Nadel, ${ }^{3} \mathrm{M}$ Cooper, ${ }^{1} \mathrm{ME}$ Garralda. ${ }^{1}$ Academic Unit of Child and Adolescent Psychiatry, Imperial College London, London, UK; ${ }^{2}$ Adolescent Assertive Outreach Team, South West London and St George's Mental Health NHS Trust, London, UK; ${ }^{3}$ St Mary's Paediatric Intensive Care Unit, Imperial College Healthcare NHS Trust, London, UK

\subsection{6/archdischild-2014-307384.432}

Background and aims Admission to paediatric intensive care (PIC) has been linked to subsequent post-traumatic stress symptoms (PTSS) in parents. This study aimed to obtain initial estimates regarding the effect of a brief intervention on parent PTSS and explore the mediating effect of baseline parental stress.

Methods Parents of children aged 4-16 years old were randomised to intervention versus treatment as usual (TAU). The intervention was delivered within six weeks of discharge from hospital and included a psycho-educational booklet and telephone call. Parents' baseline stress was measured using the Parental Stressor Scale: PICU. Parents were followed-up a median of 5 months post discharge from PICU and measures of PTSS were obtained using the Impact of Events Scale. 